\title{
The research of mobile Agent technology applied in the network management in electric grid enterprises
}

\author{
Ganghua Yao \\ Institute of Technology, East China Jiaotong University, Nanchang Jiangxi, 330100
}

Keywords: mobile Agent technology; electric grid enterprises; network management; applied research;

\begin{abstract}
Now, electric grid enterprises play an irreplaceable role in the rapid development of informationization. Mobile Agent technology, as a new part of the electric grid, improves the network management and use ratio of information. At present, share of information is needed in many industries and administrative management, so the development and application of technology are good for network environment, which provides a convenient service for people.
\end{abstract}

\section{Introduction}

China has made an extraordinary achievements in economic technology since reform and opening up. In order to conform to modernization, application of information service should be based on network in daily life. The network management system provides a favorable support for a normal network environment. At present, there is only a simple network management protocol, which manages networks in rotation from Client to Server but is limited under static state.

In the reform and development of the network management technology, the innovation is always favored. The improved mobile Agent technology based on research of technology in the last generation is much more favored by enterprises[1]. Mobile Agent technology is better for most of computers and is strong in intensity and flexible in operation and is characterized by its unique equipment and management model at the platform.

\section{Application of mobile Agent technology}

\subsection{Technical principles}

In order to realize the calculation at a large scale, the distributed technology has made a rapid development. As there is a choke point, the technology is experiencing difficulties. However, mobile Agent technology solve the technical problems of distributed technology. It realizes the independent computing and automatic mobilization in only one computer, which alleviates operation pressure by automatically transferring data to server through intermet. The technology is self-determined, intellectualized and networked, good for software development and network intellectualized management,etc. Mobile agent system's structure is shown in Fig 1.

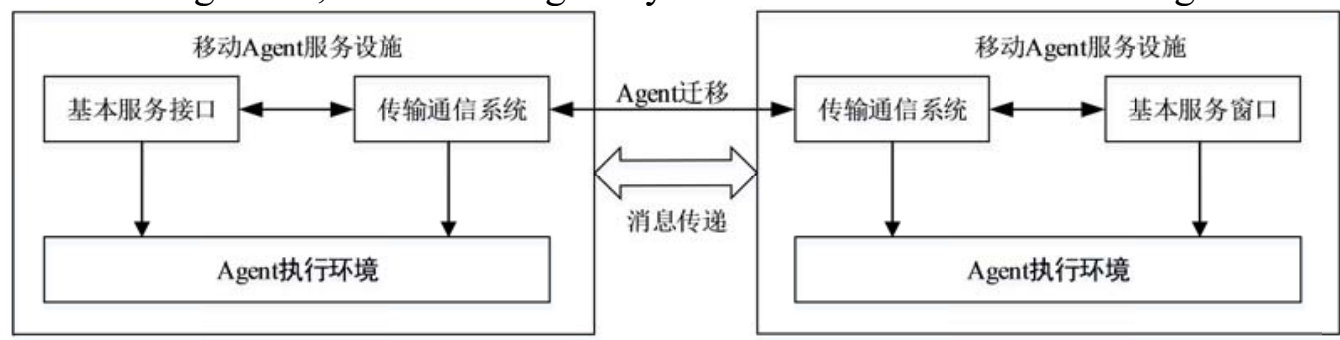

Fig 1 Mobile Agent system structure

\subsection{Mobile Agent technology and Network management}

Mobile Agent technology, as a product of the development of computer at a certain period, combines distributed and intelligent technology, solving the key technology of calculation at a large 
scale. The automatic transferring mobile Agent technology is processed by coding rather than in traditional way.

Network management is basically required at the age of informationization, which ensures the stable operation of network, improves the use ratio of network and is good for network with frequent visits.

The combination of mobile Agent technology and network management make full use of the intelligence of the technology and solve the problems of low safety and performance, providing reliable support for modern network management[2]. Its advantages are shown below.

(1) Intelligence. After solving the problem of limit in areas in traditional management, mobile Agent technology can better master the analyzed data in all around way and guard against the leakage of data, thus lessening the potential risk. Through the intelligent way of analysis and model of calculation, mobile Agent technology can fulfill the task in a more scientific and rapid way.

(2) Efficiency. As the technology can directly interacts with data, it breaks through the data transferring model and avoids the time extension caused by network failure. Only after task is fulfilled, mobile Agent technology will respond the results, which solves the problem of interaction and improves efficiency. In particular, the technology enjoys exceptional advantage in real time task monitoring.

(3) Continuity. The technology is uninterruptedly transferring and dealing with data, which guarantee the completeness and saving of key data.

(4) Cooperation. The technology possesses distributed advantage and it can cooperate among many agents at the same time, thus realizing the task of rapidity and complementing each other's advantage. By cooperation, it can improve the reliability and reduces the loss of efficiency.

\section{Mobile agent network management model}

\subsection{Inserting model}

In inserting mobile Agent model, each agent is independently produced and irrelevant with each other[3]. Agent can be transferred among different subnets and nodes by the navigating Agent.

When the tasks for subnets are the same, Agent can transfer into different subnets to fulfill the task. Inserting mobile Agent's model coding is simple, only includes codes that transfer local resources and does not include the management of subnets, as a result, it is easy to write.

Inserting mobile Agent's speed is only determined by the mobile rate of navigating Agent. It is characterized by high repetition frequency, quick mobile rate, low resources occupancy and bright development future, etc.

\subsection{Master-slave model}

In master-slave mobile Agent model, slave Agent is characterized by simple coding, bundled functions and easy-to-mobilize among different subnets. Salve agent is independent to each other. The master agent is characterized by strong function and dealing ability and is responsible for receiving Agents.

The obvious difference between master and slave agents is that master agent is responsible for common functions while slave agent is mainly responsible for core functions. Master Agent's virtual name is the same and easy to be positioned, while slave agent's virtual name is changing with tasks and mobilize rapidly in the internet.

\subsection{Agent group model}

In agent group mobile Agent model, each agent group is divided based on principles of subnets, that is to say, each agent owns its own subnet. In order to alleviate network congest, the scale of Agent is limited in a certain agent.

In the model of agent groups, the head of groups has a mobile Agent which mobilizes in the network. The head is determined by mobile Agent through selection. if the model is started and the selected head fails to react, a new head will be determined by agent groups through election. 


\subsection{Isomeric model}

In isomeric model, network management is strengthened by integration of many Agents through expert system. The model improves efficiency of network management by improving traditional network management system. As the model obviously uses the advantage of mobile Agent, it can realize the rapid and convenient network management, which indicates the wide application of isomeric model.

\section{Design of network management model based on mobile Agent}

\subsection{Model system structure}

Based on mobile Agent technology principles, a new model of network management is offered. The model can improve weaknesses in the network management such as: low in operation and real time, reduce demand for resources from network and make full use of resourc es in the computer. Mobile Agent's network management model is shown in Fig.2.

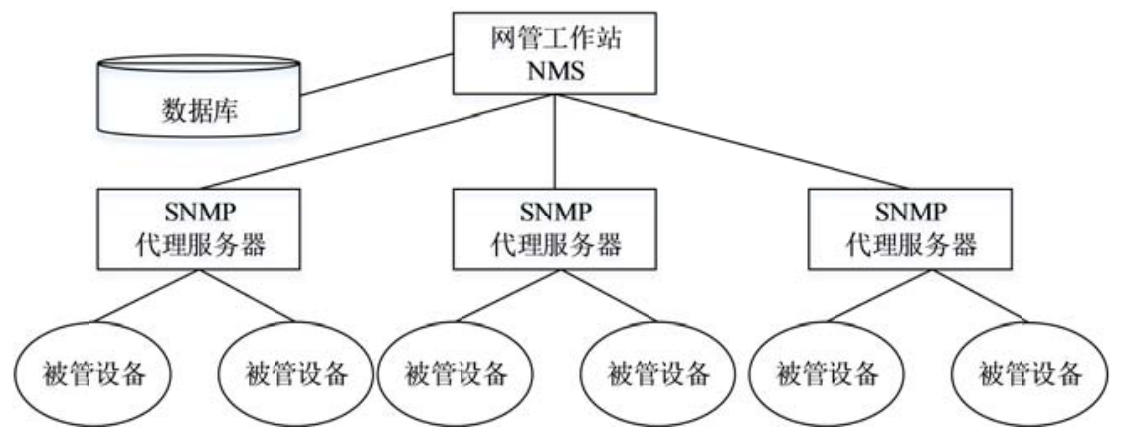

Fig 2 Mobile Agent's network management model

In addition, the environment of mobile Agent in server is the most important part and plays an important part in connecting the proceeding and following. The correction of current network management is reduced to the lowest extent in mobile Agent environment and shows the biggest advantage of mobile Agent. In model, server mainly receives mobile Agent from working station, analyzes mobile Agent, communicates with managed equipments and collects data from them.

\subsection{Model system's constitution}

In the model in Fig.2, the model mainly includes three parts: webmaster working station, SNMP agent server and managed equipments.

(1) Webmaster working station: webmaster working station is the core equipment in network management, produces different Agent based on different tasks and can send and deal with data of mobile Agent in no time. In some mobile agent platforms, webmaster working station can set up coding management's server and strengthens coding management for mobile Agent. NMS's functions is shown in Fig.3.

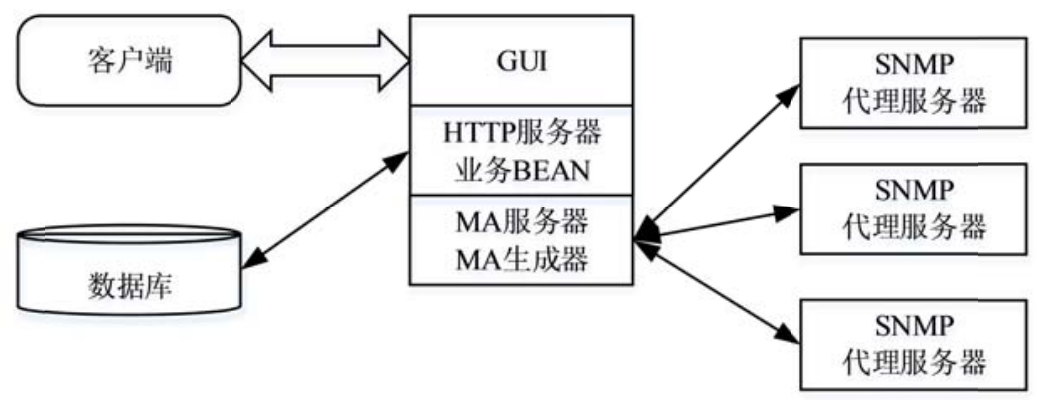

Fig. 3 NMS function drawing

(2) SNMP agent server: SNMP agent server is able to mobilize Agent among subnets and offer reception, copy and verification, etc. Mobile Agent server set up the platform for Agent, which 
makes it easy for mobile Agent to acquire data in data base, thus ensuring the completion of task. SNMP's management entity can transfer data with mobile Agent through protocol, thus acquiring the variation value of managed equipment.

(3) Managed equipment: managed equipment is the final performer of orders. It can be any equipment in network management, such as: interchanger, computer, etc. The managed equipment in the model is similar to the one in traditional network management, which mainly receives and executes orders, visits data base and send orders to SNMP agent servers if necessary.

\subsection{Network management calculation and its realization}

Network topological information is very important for network management. Through accurate topological information, staffs can understand whether the equipment is correctly set up and find the problems in the internet and monitors the real time operation of networks. At present, transferring ethernet gradually replaces the traditional sharing ethernet. The transferring one can reduce the network congestion caused by sharing, improves the safety and the complexity of connection among network equipments. In the design of the model above, a kind of network topological searching calculation is produced, which makes research in traditional calculation and combines with mobile Agent technology.

In the topological calculation of spanning tree protocol, each equipment sends and acquires information from terminals. Based on the comparative analysis of information above, optimal results is reserved. Only after acquiring optimal results, the position and function of spanning trees and bridges can be determined.

\section{System design testing}

The platform for testing system is Windows 7 operation system and the used software is IBM's Aglets, Advent's AdventNetSNMP and mysql's database. Java is needed as Aglets need it. Mobile Agent and SNMP communicate with each other by data base and transfer data by mysql. In the test, network's performance is shown by monitoring time extension, handling capacity and pocket loss probability,etc.

(1) Time extension: network time extension mainly includes single and round time extensions. The commonly used one is round time extension, which means the time of sending and receiving the data.

(2) Handling capacity: it means the transferring speed of information in the internet, that is to say, the total speed of data or the speed of a certain type of data. At present, the calculation method of handling capacity is mainly selecting related variations of important equipments or analyzing data package. The design in the text chooses the former way to calculate handling capacity.

(3) Loss package rate: in the internet transferring, data is easily lost so the loss package means the degree of loss. If the data is lost, the equipment will resend data, which improves the burden of internet.

\section{Analysis of test results in the system}

Based on the simulated tests in the design above, testing is conducted by software. According to the results, based on the comparative analysis of time extension between the two types, advantages and disadvantages of them are elaborated. 


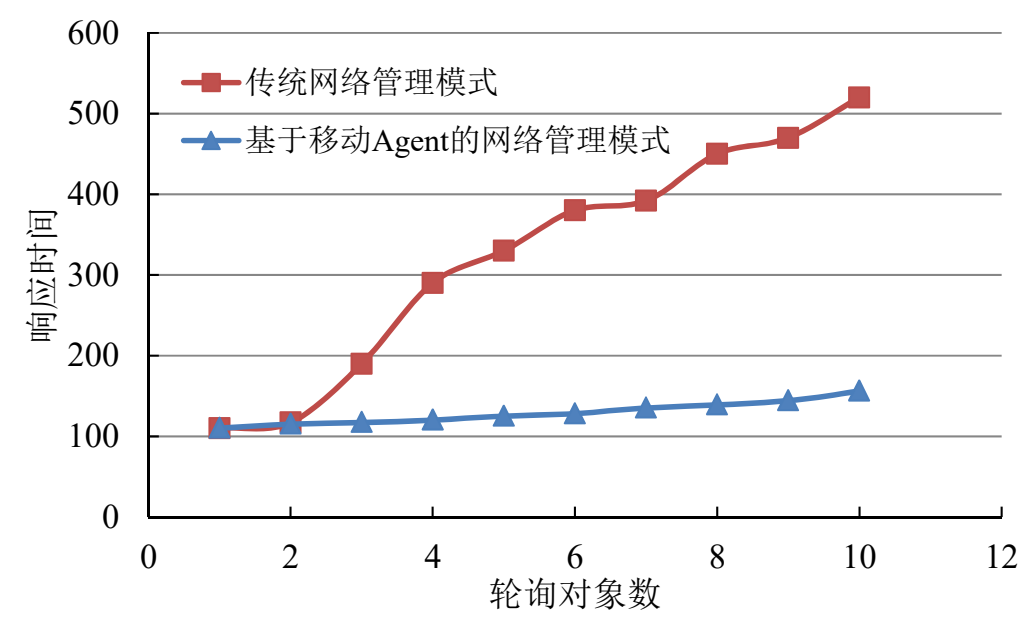

Fig.4 Comparison between two types of network managements

In Fig.4, when polling 1 and 2, the difference between the time extension of the two types is not very big; when the number of polling increases, the time extension based on mobile Agent network management is much shorter than traditional one. Based on testing results, mobile Agent network management model is much better than traditional one.

\section{Conclusion}

The potential of electric grid is big, which may be faced with bigger and more complicated network management. Based on the network management model of Agent and comparison with the traditional model, mobile Agent can improve the efficiency of the network management and ensure the stable and safe operation of electric grid enterprises in the future.

\section{References}

[1] Ma Zhili. The research of mobile Agent technology applied in the electric grid enterprises' network management[J]. electric information and communication technology, 2015,13(2):123-126.

[2] Zhou Jia. The application of mobile Agent technology in the network management in enterprises. Digital technology and application, 2015, (8): 155-156

[3] Fan Jing. The research of inserting mobile Agent internet management models[J]. Journal of Yunnan Minzu University(natural science edition),2006,15(3):242-246. 